\title{
The Analysis of Exhaust Gas Thermal Energy Recovery Through a TEG Generator in City Traffic Conditions Reproduced on a Dynamic Engine Test Bed
}

\author{
JERZY MERKISZ, ${ }^{1}$ PAWEL FUC, ${ }^{1}$ PIOTR LIJEWSKI, ${ }^{1}$ \\ ANDRZEJ ZIOLKOWSKI, ${ }^{1,3,4}$ and KRZYSZTOF T. WOJCIECHOWSKI ${ }^{2}$
}

\begin{abstract}
1.-Faculty of Machines and Transport, Institute of Combustion Engines and Transport, Poznan University of Technology, Piotrowo St. 3, 60-965 Poznan, Poland. 2.-Thermoelectric Research Laboratory, Department of Inorganic Chemistry, Faculty of Materials Science and Ceramics, AGH University of Science and Technology, Mickiewicza Ave. 30, 30-059 Cracow, Poland. 3.-e-mail: andrzej.j.ziolkowski@put.poznan.pl.4.—e-mail: andrzej.wo.ziolkowski@doctorate.put.poznan.pl
\end{abstract}

\begin{abstract}
We present an analysis of thermal energy recovery through a proprietary thermoelectric generator (TEG) in an actual vehicle driving cycle reproduced on a dynamic engine test bed. The tests were performed on a $1.3-\mathrm{L} 66-\mathrm{kW}$ diesel engine. The TEG was fitted in the vehicle exhaust system. In order to assess the thermal energy losses in the exhaust system, advanced portable emission measurement system research tools were used, such as Semtech DS by Sensors. Aside from the exhaust emissions, the said analyzer measures the exhaust mass flow and exhaust temperature, vehicle driving parameters and reads and records the engine parameters. The difficulty related to the energy recovery measurements under actual traffic conditions, particularly when passenger vehicles and TEGs are used, spurred the authors to develop a proprietary method of transposing the actual driving cycle as a function $V=\mathrm{f}(t)$ onto the engine test bed, opn which the driving profile, previously recorded in the city traffic, was reproduced. The length of the cycle was $12.6 \mathrm{~km}$. Along with the motion parameters, the authors reproduced the parameters of the vehicle and its transmission. The adopted methodology enabled high repeatability of the research trials while still ensuring engine dynamic states occurring in the city traffic.
\end{abstract}

Key words: Waste heat recovery, TEG, PEMS, energy conversion

\section{INTRODUCTION}

A reduction of energy consumption of modern vehicle powertrains is one of the main factors determining their development. ${ }^{1-3}$ Reduction of energy consumption should be construed as that which involves all methods to achieve the desired result (reduction of consumption of energy by a vehicle). In modern vehicles, this is realized through modifications in the design of piston engines and their aggregates, the application of thermal energy recuperation systems, and the use of all kinds of

(Received July 29, 2014; accepted November 6, 2014; published online December 4, 2014) additional systems aimed at a reduction in fuel consumption. Modern piston engines are characterized by much higher volumetric power indexes than engines of older generations. This allows a reduction of the engine displacement, which, in many cases, entails a reduction of the number of cylinders. This reduces friction losses in the crankshaft and, particularly, piston assemblies. ${ }^{4,5}$ It also leads to a reduction in fuel consumption. Another method to reduce energy consumption is the possibility of energy recuperation from the exhaust gas. ${ }^{6,7}$ In Dingel et al., 8 a classification of thermal energy recuperation methods that are currently used in motor vehicles has been carried out. The most popular method of energy recuperation is 
engine supercharging realized through turbocharger systems fitted in the engine exhaust system. Almost every diesel engine is fitted with this type of system. Also, in spark ignition engines, turbocharger systems are used increasingly, following the process of downsizing. ${ }^{4,5}$ Beside the methods of conversion of thermal energy into mechanical energy, systems that convert thermal energy into electrical energy are applied. All types of electric generators (turbogenerators, TEG generators, etc.) belong to this category. This type of system can be successfully applied in vehicles fitted with both conventional and hybrid (combustion engine and electric motor) powertrains. ${ }^{9,10}$ The paper presents an analysis of the influence of the application of a prototype TEG generator in the engine exhaust system of a diesel engine. The research was performed on an engine test bed fitted with a dynamic brake (based on a proprietary test procedure). The test was a reproduction of the actual driving profile recorded during typical urban and suburban road conditions utilizing PEMS equipment. The reproduced test also included clutch and transmission parameters of the powertrain, design parameters of the vehicle and the driver's behavior.

\section{DETERMINING THE ACTUAL DRIVING PROFILE OF A VEHICLE}

\section{Research Method}

In order to determine the actual driving profile as a function $V=\mathrm{f}(t)$, tests under actual operating conditions were performed using advanced research tools (PEMS). The tests were performed on a test route whose total length was $12.6 \mathrm{~km}$. When selecting the test route, the authors aimed at reproducing typical urban (involving frequent vehicle stops) and suburban conditions. The selected test route met the said requirements. The 'urban' part included highly congested streets and involved a variety of intersections. The 'suburban' part was a portion of the national road 92-one of the main eastern entrance roads to the Poznan agglomeration. Such varied road conditions allow conducting analyses under a wide range of speeds and varied accelerations. This influences the engine effective parameters, which is directly translated into the amount of thermal energy of the exhaust gas.

The research object was a minivan fitted with a $1.3-\mathrm{dm}^{3} 66-\mathrm{kW}$ diesel engine. The vehicle curb weight was $1275 \mathrm{~kg}$ (Table I). An identical engine was tested on the AVL DynoRoad 120 dynamic test bed.

The authors used equipment for exhaust emissions measurement under actual operating conditions (PEMS) Semtech DS made by Sensors (Fig. 1a). It measures and records the following parameters $^{11,12}$ :

(a) Concentrations of $\mathrm{CO}$ and $\mathrm{CO}_{2}$ (NDIR analyzerNon-Dispersive Infrared), $\mathrm{NO}_{x}=\mathrm{NO}+\mathrm{NO}_{2}$

\section{Table I. Specifications of the tested vehicle}

\begin{tabular}{lc}
\hline Parameter & Value \\
\hline $\begin{array}{l}\text { Powertrain } \\
\text { Ignition }\end{array}$ & Self-ignition \\
Number of cylinders/ & $4 / 4$ \\
valves per cylinder & $1.3 \mathrm{dm}^{3}$ \\
Displacement & $66 \mathrm{~kW} @ 4000 \mathrm{rpm}$ \\
Maximum power & $200 \mathrm{Nm} @ 1750 \mathrm{rpm}$ \\
Maximum torque & Common rail \\
Injection system & Euro 4 \\
Emission standard & DOC \\
After-treatment & Manual \\
Transmission & \\
Gear ratios & $3.91: 1$ \\
Gear I & $2.24: 1$ \\
Gear II & $1.44: 1$ \\
Gear III & $1.03: 1$ \\
Gear IV & $0.77: 1$ \\
Gear V & \\
Vehicle & \\
Dimensions & $3930 \mathrm{~mm}$ \\
Length & $1698 \mathrm{~mm}$ \\
Width & $1600 \mathrm{~mm}$ \\
Height & \\
Wheelbase & $1275 \mathrm{~kg}$ \\
Curb weight & 5 \\
Number of doors & 0.31 \\
Drag coefficient & $173 \mathrm{~km} / \mathrm{h}$ \\
Maximum speed & \\
\hline
\end{tabular}

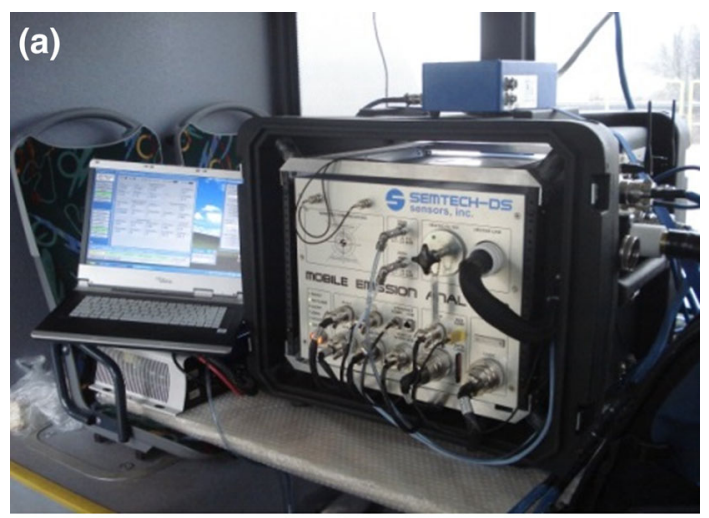

(b)

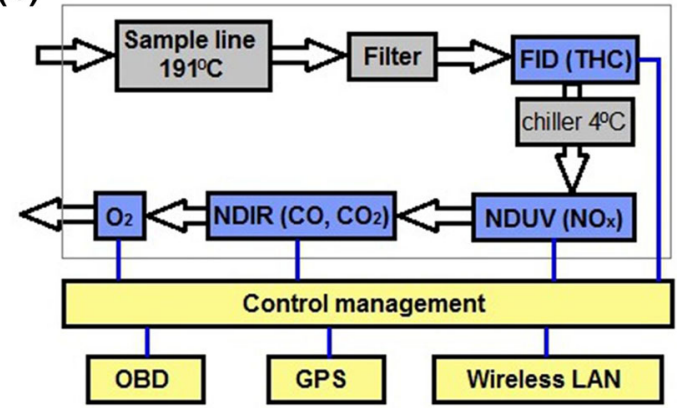

Fig. 1. Semtech DS (a) and its operational diagram (b). 
(NDUV analyzer-Non-Dispersive Ultraviolet), THC (FID analyzer-Flame Ionization Detector), $\mathrm{O}_{2}$ (electrochemical sensor);

(b) Exhaust gas mass flow-Pitot tube flow sensor,

(c) Ambient conditions-ambient pressure, temperature, humidity;

(d) Vehicle instantaneous speed and position-GPS system;

(e) Data taken directly from the vehicle OBD.

The exhaust gas is introduced into the analyzer through a probe maintaining the temperature of $191^{\circ} \mathrm{C}$ (Fig. 1b). Then, the particulate matter is filtered out (diesel engines) and the exhaust gas is directed to the flame-ionizing detector where the concentration of THC (total hydrocarbons) is measured. The exhaust gas is then chilled to the temperature of $4^{\circ} \mathrm{C}$ and the measurement of the concentration of $\mathrm{NO}_{x}, \mathrm{CO}, \mathrm{CO}_{2}$ and $\mathrm{O}_{2}$ follows.

For the measurement of the exhaust gas mass flow, a mass flow meter was used that was part of the portable PEMS exhaust gas analyzer (Semtech DS). The mass flow rate of the exhaust gas was determined based on the flow continuity and Bernoulli equations using the Pitot tube ${ }^{13}$ :

$$
\dot{m}=K(R e) \times A \sqrt{\rho \times \Delta P}
$$

where $\dot{m}$ is the exhaust mass flow, $A$ the physical cross-section of the area of the flow tube assembly, $K(R e)$ the discharge coefficient for the flow tube assembly as a function of the Reynolds Number, $\rho$ the density of the exhaust gas, and $\Delta P$ the difference between $P_{\mathrm{HIGH}}$ and $P_{\text {LOW. }}$.

Figure 2 presents Semtech EFM and the crosssection of the Semtech EFM averaging Pitot tube flow sensor.

The exhaust density is determined by the Semtech portable exhaust emissions analyzer software based on the exhaust temperature upstream of the flow tube assembly and the molecular mass of the exhaust gas. Semtech determines the molecular mass based on the measured constituent concentrations. Semtech then applies a correction to the raw flow readings based on the calculated density. As noted above, one difficulty with flow meters based on differential pressure $(\Delta P)$ is a limited dynamic range. Because of the square-root relationship, large changes in $\Delta P$ reflect small changes in the flow. To overcome this problem, Semtech EFM has incorporated four $\Delta P$ sensors with ranges that span more than two orders of magnitude. Each sensor is selected specifically for certain portions of the total flow range. With this arrangement, vehicle exhaust can be measured accurately over its entire range from idle to maximum flow. ${ }^{13}$

\section{Results of the Measurements: Vehicle Driving Profile}

The measurements were performed in the afternoon hours on a typical weekday. This aimed at
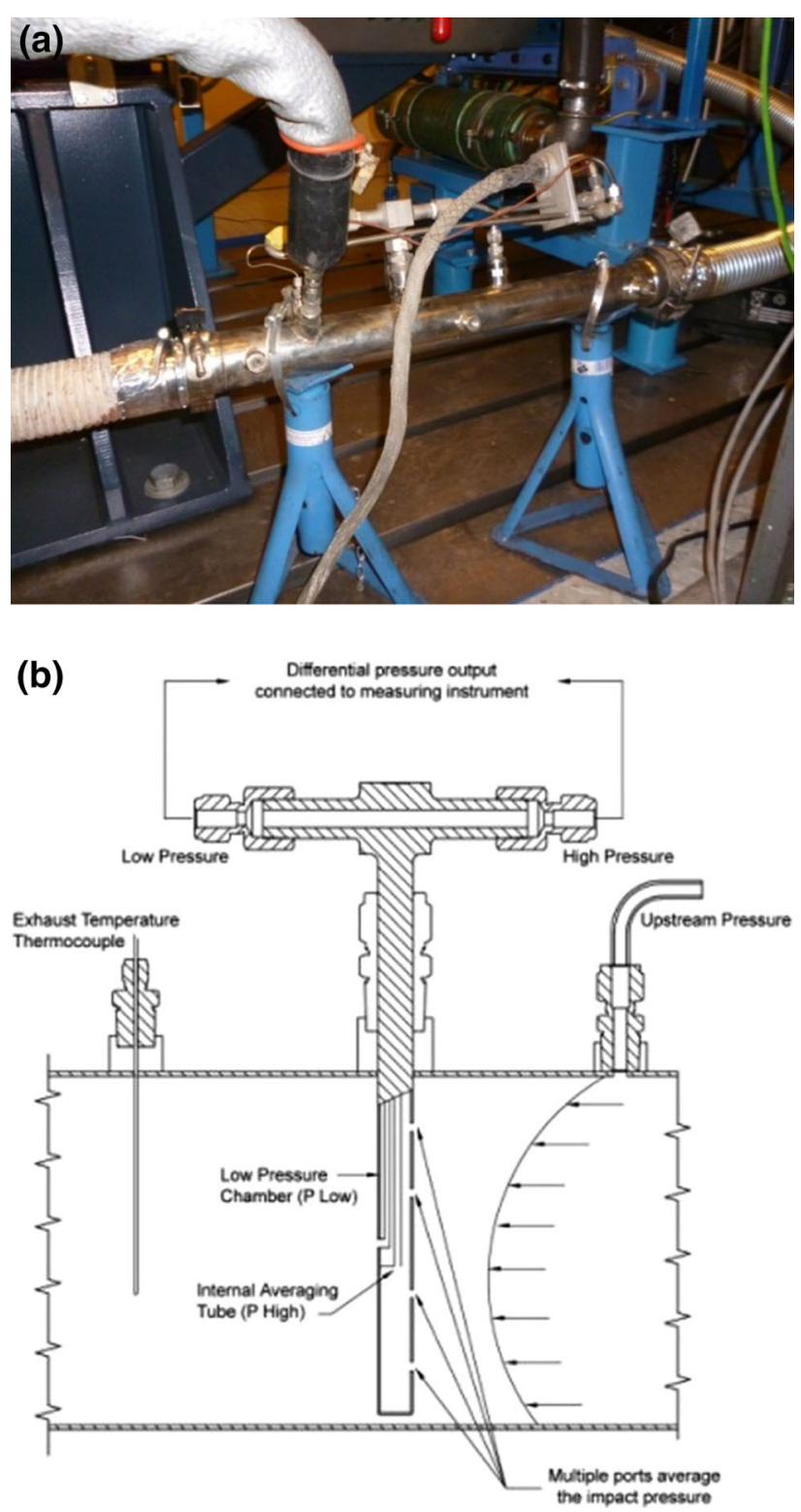

Fig. 2. View of Semtech EFM (a) and flow tube assembly crosssection (b). ${ }^{13}$

performing the tests under the conditions representative of the Poznan agglomeration traffic rate and typical of majority of cities of the population exceeding 500,000. The test route was a combination of urban and suburban traffic conditions. The driving profile was a reproduction of the test route and is expressed with a function of speed $V=\mathrm{f}(t)$ and acceleration $a=\mathrm{f}(t)$. From the two, we know that the vehicle had significant speed variations and, consequently, varied accelerations because of the intersections on the test route (Fig. 3a). The test run took $1441 \mathrm{~s}$ and the vehicle had an average speed of $31.7 \mathrm{~km} / \mathrm{h}$. The maximum speed did not exceed $80 \mathrm{~km} / \mathrm{h}$, which also resulted from high traffic congestion on the test day. The share of vehicle stationary phase was $13.8 \%$ of the entire 
vehicle run, and the acceleration phase was $44.4 \%$ (Fig. 3b).

The share of the test run at a steady speed was negligibly low. Upon referring the measurement conditions to the engine parameters, the authors have found that it operated mainly at a medium load at the speed of 1200-2400 rpm (Fig. 4).

Hence, the mass flow and temperature of the exhaust gas did not have high values, as would have been in the case had the engine operated at maximum loads. The above facts are a confirmation of the test objective, which was a reproduction of the typical urban and suburban driving conditions.

\section{REPRODUCTION OF THE ACTUAL DRIVING CYCLE OF A VEHICLE ON AN ENGINE TEST BED FITTED WITH A DYNAMIC BRAKE}

\section{Engine Stand for Testing Under Dynamic Conditions AVL DynoRoad 120}

AVL DynoRoad engine test bed is designed for testing piston combustion engines under stationary, non-stationary and dynamic conditions. The test stand is composed of a dynamic brake (three-phase cage-type electric machine), coupling shaft (engine brake) as well as fuel conditioning and feed systems (Fig. 5). The test stand is installed on a special plate

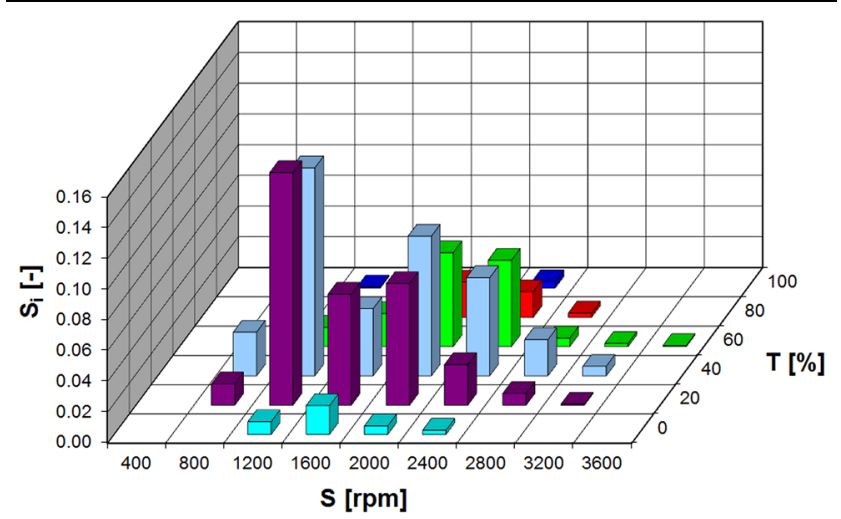

Fig. 4. Time share characteristics obtained in the engine speed and torque intervals.

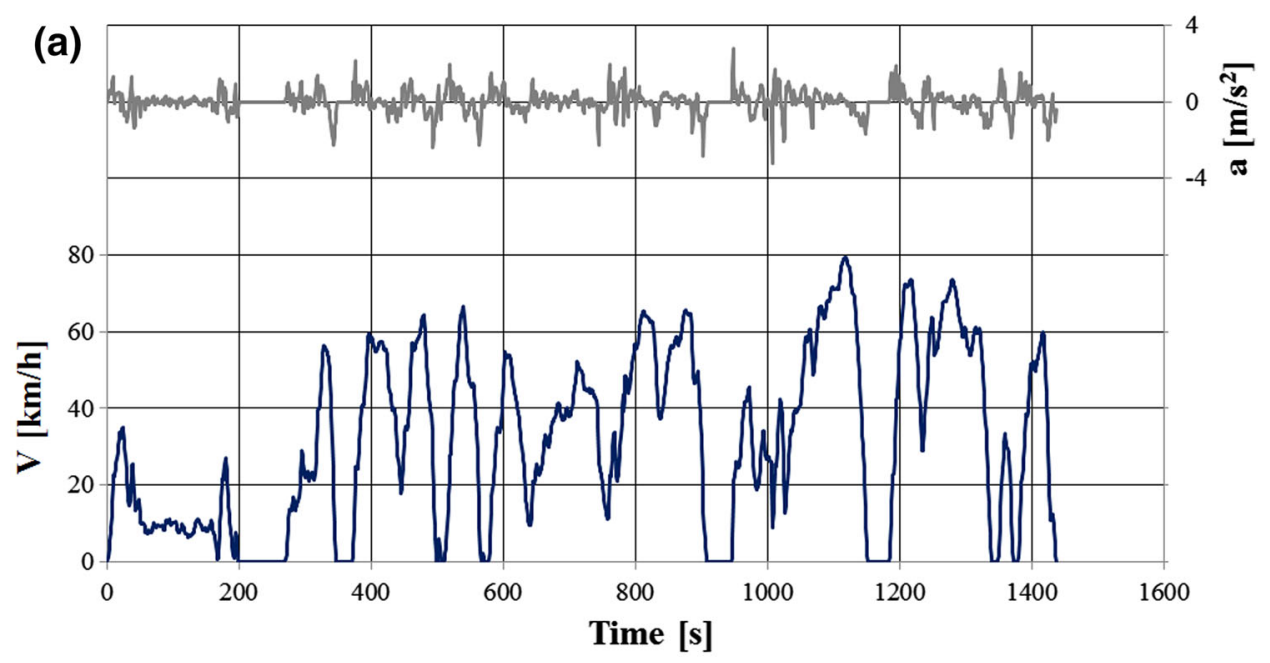

(b)

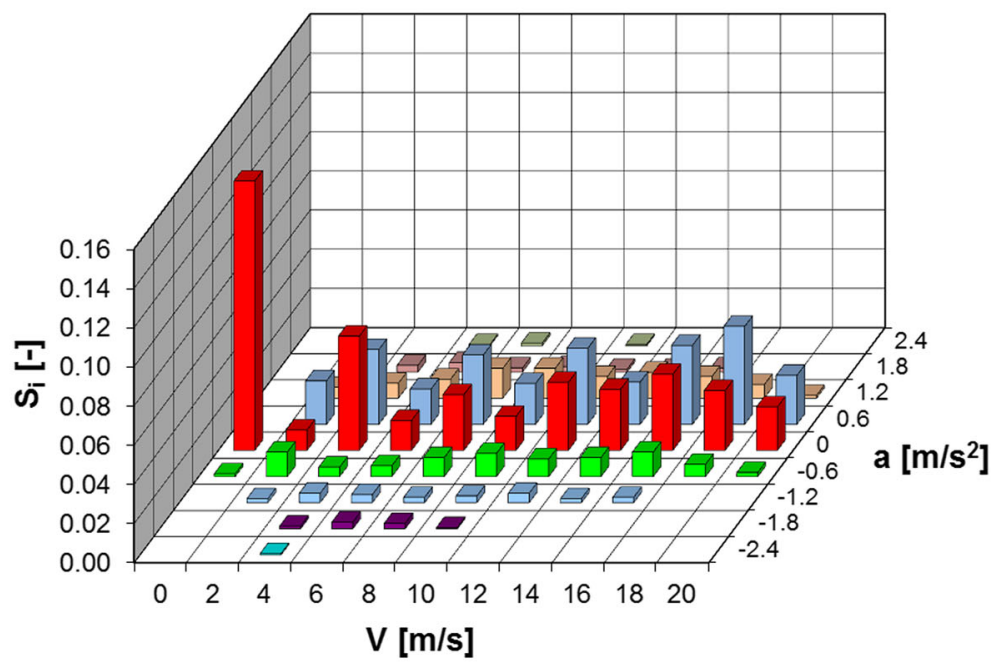

Fig. 3. Speed and acceleration tracings (a) and operating time share during the road test (b). 

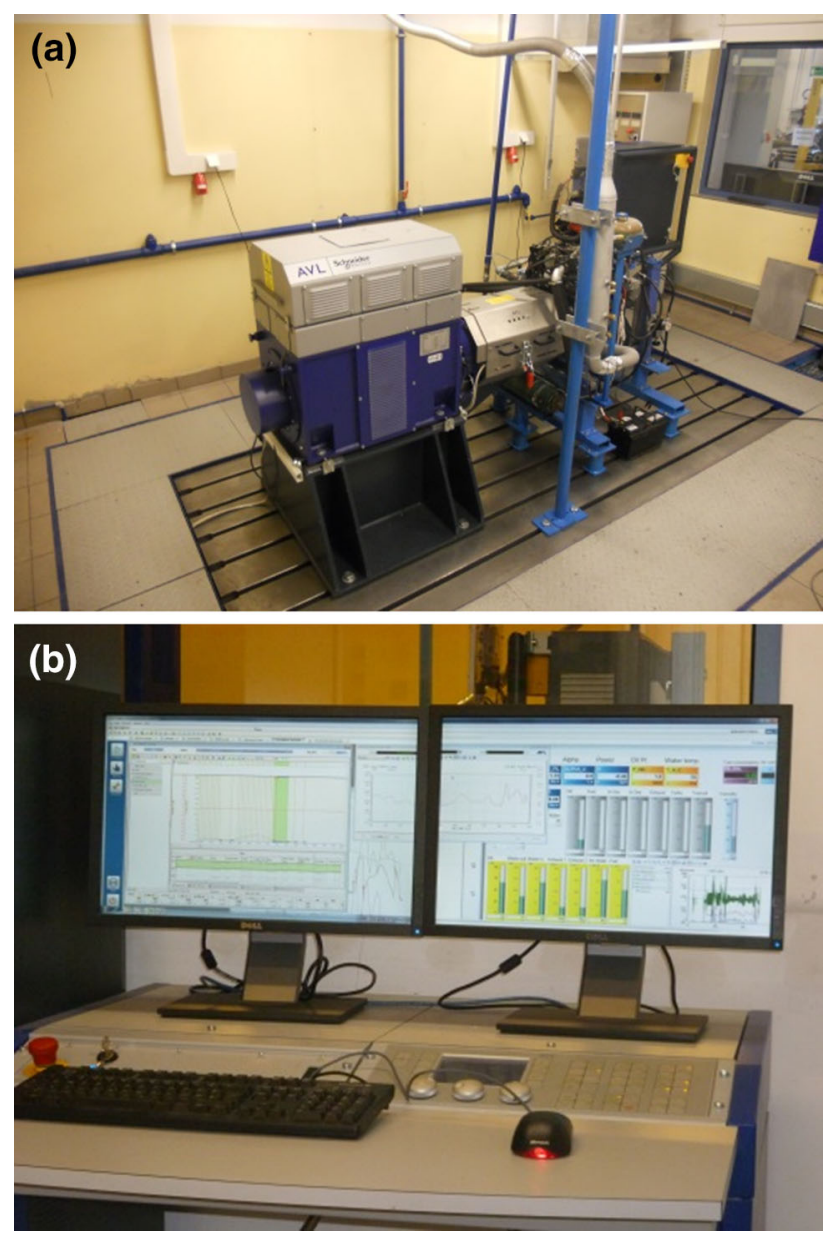

Fig. 5. AVL DynoRoad 120 kW: (a) dynamic engine dynamometer, (b) control panel.

damped with pneumatic cushions to compensate all type of vibrations generated during the measurement. The test stand, aside from the main systems, also has a variety of sensors measuring temperature, pressure, engine speed etc.

The test stand is controlled with test bed and measurement automatics (PUMA Open). It is an advanced system enabling automated control of the test stand. This is realized through implemented test procedures and monitoring of all parameters of engine and test stand operation. The system enables determining critical values of individual parameters upon excess of which the test stand goes into a limp mode and shuts down the engine. This safeguards against uncontrolled malfunctions.

\section{Simulation Software ISAC}

ISAC 400 software is designed to simulate the vehicle driving profile, traffic conditions, vehicle design and driver's behavior. The software is used in combination with engine monitoring and control system and PUMA Open on engine test beds fitted with a high-tech dynamic engine brake made by

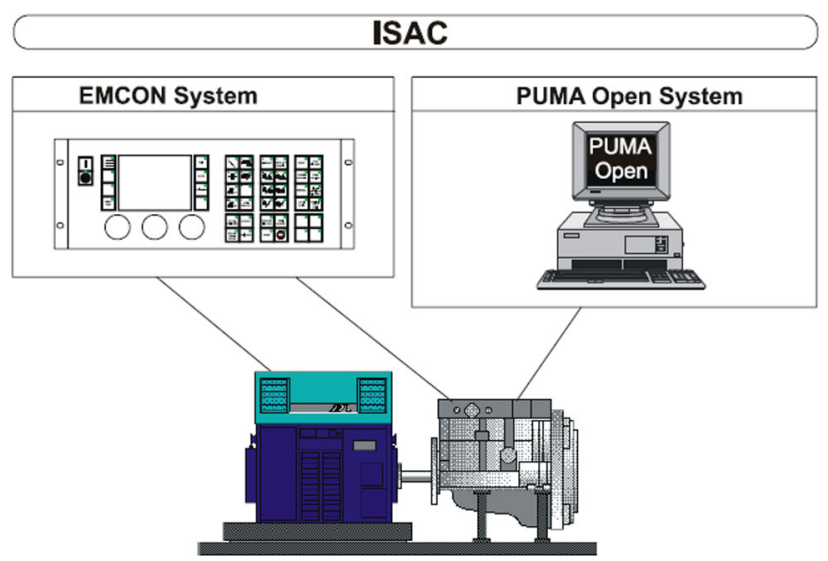

Fig. 6. Structure of the ISAC software. ${ }^{14}$

AVL (Fig. 6). ISAC 400 allows performing tests that were thus far only possible to realize on chassis dynamometers where the entire vehicle had to be tested. The advantages of the software are ${ }^{14}$ :

- realistic load tests in early development stages;

- the engine does not have to be fitted into the vehicle

- better engine accessibility (fixing sophisticated measuring equipment),

- reproducibility of the tests, reduced dependence on road conditions and the constitution of the test driver,

- all varieties of driver and vehicle behavior can be simulated (from sporty to economic drivers),

- statutory test cycles (FTP75 and NEDC) can be carried out on the engine test bed,

- the advantages described above help reduce the costs of development,

- simultaneous development of vehicle and engine prototypes.

In ISAC 400, NEDC and FTP 75 homologation test profiles are available. If a different profile of a driving cycle is recreated, it is necessary to model its course. To this end, each step of the test needs to be defined via a step sequence editor (SSQ). Figure 7 shows an example configuration window of individual steps of a new research cycle.

\section{Reproduction of the Vehicle Driving Profile on the Dynamic Brake Test Stand}

Using the SSQ, the recorded driving profile of the vehicle expressed as a function $V=\mathrm{f}(t)$ along with the road gradient was uploaded to the ISAC 400 software. Additionally, the vehicle speed profile was integrated with the gear ratios of the transmission in the simulation software, which was necessary for a proper operation on the test stand. The parameters of the tested object were also uploaded to the system. Using the implemented speed profile a fully automatic test was developed composed of block elements of the following configuration: 


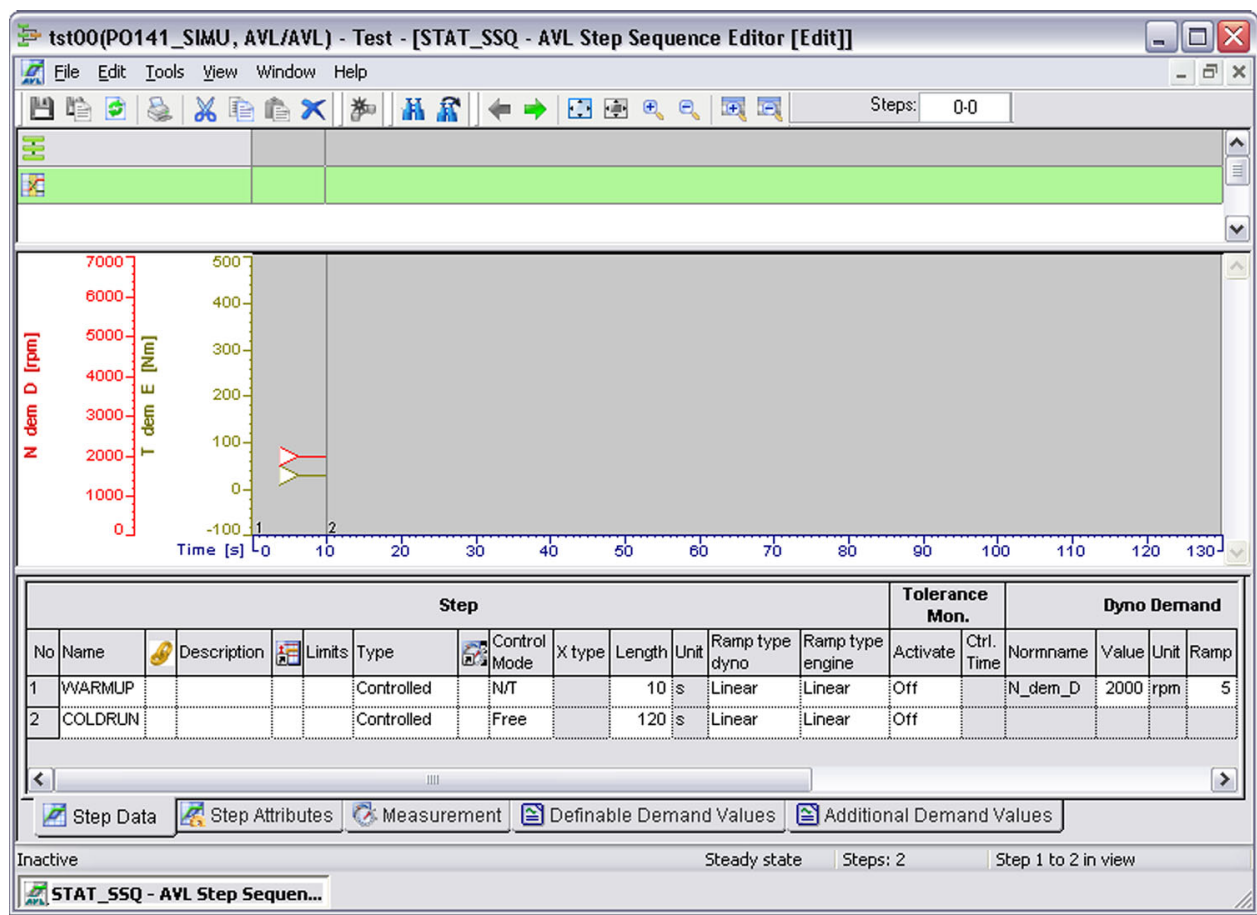

Fig. 7. View of the test sequence in the step sequence editor. ${ }^{15}$

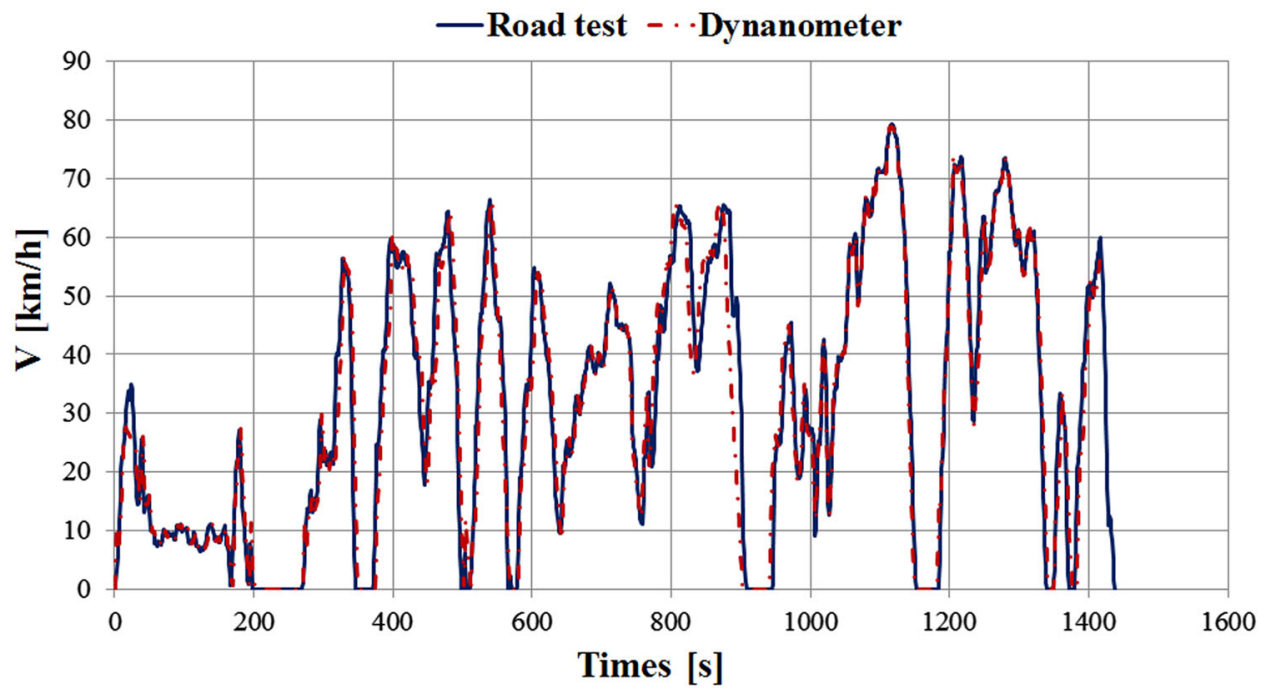

Fig. 8. Comparison of the speed profiles in the road test and on the test stand.

(a) engine start,

(b) engine warm-up to $60^{\circ} \mathrm{C}$ (coolant temperature),

(c) parameter recording on (PUMA),

(d) realization of the programmed vehicle speed profile,

(e) parameter recording off,

(f) engine cooling down,

(g) engine shut off.

A similarity of the speed profile recorded in the road test to that obtained on the test stand was observed (Fig. 8). Only in individual points of work were differences spotted. This may result from the characteristics of the transmission and clutch operation. In the road test, the use of clutch by the driver is practically unrepeatable, while on the test stand the characteristics of the clutch and transmission operation is preset and repeatable for each gear change. This, however, does not significantly influence the difference between the average speeds in the road test and those reproduced on the test stand-they were 31.8 and $31.7 \mathrm{~km} / \mathrm{h}$, respectively.

The fact of successful reproduction of the actual driving profile of a vehicle on an engine test bed is confirmed by the linear relation between the actual 


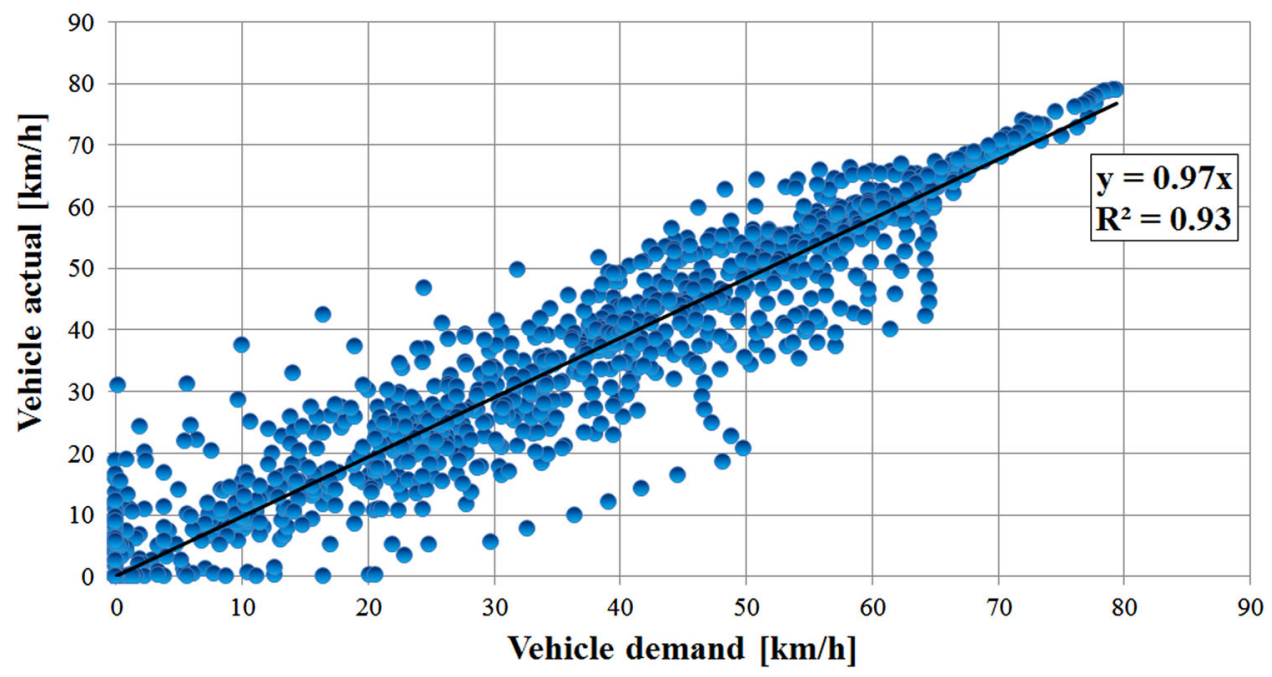

Fig. 9. Correlation of the speed in the road test and that obtained on the test stand.

and the reproduced profiles, whose coefficient of determination is $R^{2}=0.93$ (Fig. 9). From the statistical analysis, we know that obtaining a coefficient in the range of 0.9-1.0 confirms a very good compatibility of the model with the exogenous variable.

\section{TESTS ON THERMAL ENERGY RECUPERATION FROM THE EXHAUST GAS SYSTEM PERFORMED ON THE AVL DYNOROAD 120 TEST STAND USING A THERMOELECTRIC GENERATOR (TEG)}

\section{Testing Procedure}

Analyses of thermal energy recuperation from the exhaust gas were performed on the AVL DynoRoad 120 test stand with a test procedure described in section 3.3. In the exhaust system of the tested engine (composed of the same elements as the exhaust system of an actual vehicle; Fig. 10), a TEG of own design was applied.

We can distinguish two main components in the design of TEGs: the heat exchanger and the thermoelectric modules, which are a fundamental component of a TEG. The heat is absorbed from hot gas by a heat exchanger HX and transferred to the thermoelectric modules. The heat flux passing through the TE modules is partly converted to electric energy, and the rest of the heat is dissipated to the heat sinks (coolers). While designing a TEG system, the geometry of the heat exchanger and the type of modules must be selected depending on the temperature and mass flow of the exhaust gas. Owing to its simple design and lack of moving parts, a TEG generator has a number of advantages such as: high durability and reliability, low mass or noiseless operation. TEG generators are applied in maintenance-free devices such as space probes (e.g., Voyager missions, Curiosity rover), weather stations or military equipment. Figure 11 presents drawings of the TEG prototype.

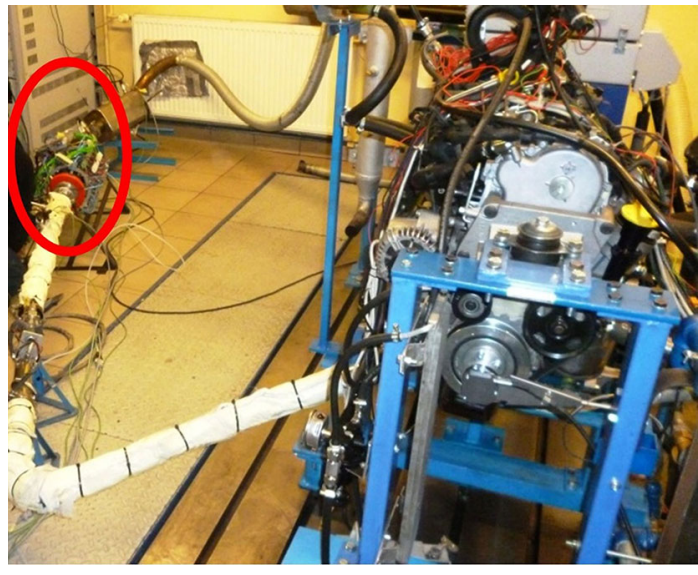

Fig. 10. View of the test stand with the TEG generator installed (circled).

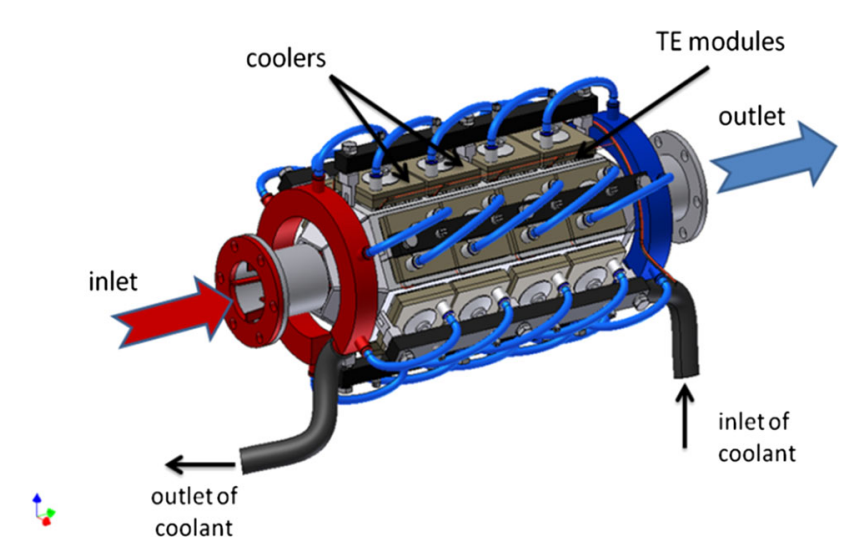

Fig. 11. Schematic view of the thermoelectric generator TEG.

The heat exchanger was made of aluminium (high thermal conductivity, low specific weight and relatively good resistance to exhaust gas-induced corrosion in temperatures of up to $300^{\circ} \mathrm{C}$.) 


\section{Table II. Parameters of the TEG generator}

\begin{tabular}{lcc}
\hline Parameters & Value \\
\cline { 1 - 1 } Total dimensions & $280 \mathrm{~mm} \times 555 \mathrm{~mm}$ \\
Dimensions of the heat exchanger & $110 \mathrm{~mm} \times 311 \mathrm{~mm}$ \\
Inner area of the heat exchange & $0.574 \mathrm{~m}^{2}$ \\
Material of the heat exchanger & Aluminium alloy \\
Number of TE modules & 24 \\
Rated power of a single TE module & $7 \mathrm{~W}\left(\mathrm{~T}_{\mathrm{c}}=50^{\circ} \mathrm{C}\right.$, \\
Total rated power of TE modules & $\left.T_{\mathrm{H}}=175^{\circ} \mathrm{C}\right)$ \\
$U_{\text {oc voltage }\left(T_{\text {in }}=250^{\circ} \mathrm{C}\right)}$ & $168 \mathrm{~W}$ \\
Maximum operating & $280 \mathrm{~V}$ \\
temperature of TE modules & $200^{\circ} \mathrm{C}$ \\
Total mass & \\
\hline
\end{tabular}

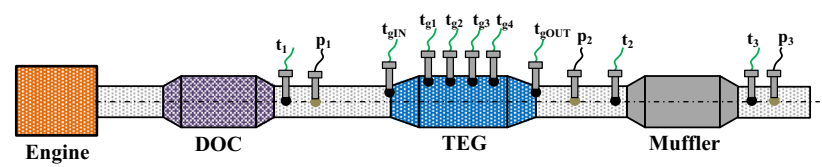

Fig. 12. Location of the exhaust gas temperature and pressure measurement points.
In order to enhance the heat transfer, longitudinal fins were used on the entire length of the HX chamber. To improve temperature uniformity along the exchanger, which was a problem in our previous prototype, variable height of the fins was used. The generator contains 24 commercial $\mathrm{TE}$ modules based on $\mathrm{Bi}_{2} \mathrm{Te}_{3}-\mathrm{Sb}_{2} \mathrm{Te}_{3}$ thermoelectric materials, ${ }^{16}$ mounted between the heat exchanger and the coolers. Each module has a separate liquid cooler fixed with a binding screw, which simultaneously tightens the module to the heat exchanger (Table II). The coolers can use water or typical automotive coolants e.g. from an external cooling system of the car engine.

For fitting convenience the TEG generator was located at the end of the exhaust system, upstream of the muffler. The exhaust system was covered with special insulation that was to reduce the exhaust gas chilling lengthwise in the system. During the tests, measurements of the following parameters of the exhaust system and its components were performed:

(a) exhaust gas mass flow-Semtech DS,

(b) exhaust gas temperature and pressure-schematics shown in Fig. 12,
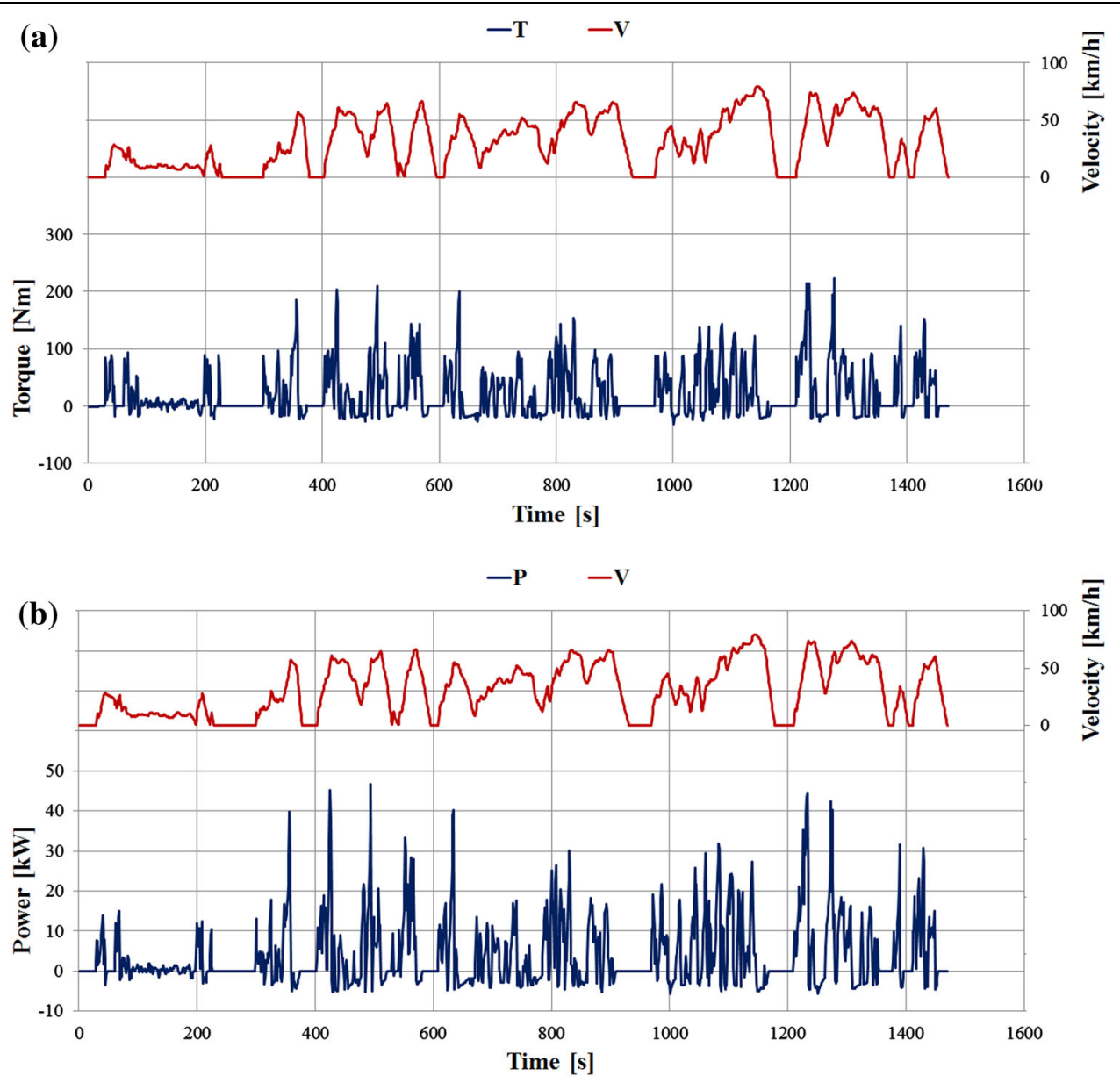

Fig. 13. Tracings of the engine torque (a), power (b) and speeds of the reproduced vehicle. 
(c) of the TEG generator:

- Temperature inside the generator at four points $\left(t_{\mathrm{g} 1}, t_{\mathrm{g} 2}, t_{\mathrm{g} 3}, t_{\mathrm{g} 4}\right)$, at the inlet and outlet $\left(t_{\mathrm{gin}}, t_{\mathrm{gout}}\right)$,

- Temperature of the coolant at the inlet and outlet from the generator cooler,

- TEG Voltage and Current.

Beside the above, the parameters of the engine were also measured (engine speed, load, fuel consumption, etc.), driving parameters of the simulated vehicle and test stand ambient conditions (temperature, pressure and humidity).

\section{Results and Discussion}

During the tests performed on the engine test bed (AVL DynoRoad 120) the engine produced a maximum torque of $213 \mathrm{Nm}$ and power of $46 \mathrm{~kW}$ (Fig. 13). These values were recorded during the maximum speed growth (acceleration of the reproduced vehicle). Negative torque and power were also recorded, which means that, in the moment that engine braking occurred, the engine brake drove the engine. The average values of both parameters, including the negative ones, were as follows: $T=21.4 \mathrm{Nm}$ and $P=3.9 \mathrm{~kW}$. Such low values of the engine average effective power and a high share of engine braking (30\%) prove a relatively low energy consumption of the driving cycle, which is why the outstanding parameters were averaged with a 10-s resolution to better depict the obtained data.

A relatively low energy consumption of the test run was directly reflected in the thermodynamic parameters of the exhaust gas, its temperature in particular. The greatest values of temperature at all measurement points occurred from the $350 \mathrm{~s}$ of the test onwards and mainly resulted from higher vehicle speed of this phase. In the first phase of the test, the vehicle speed did not exceed $32 \mathrm{~km} / \mathrm{h}$. The maximum temperature value occurred for the first measurement point and was $t_{1}=224.2^{\circ} \mathrm{C}$ (Fig. 14).
The temperature of exhaust gas at the inlet to the TEG generator was much lower and was at its maximum $t_{\mathrm{gIN}}=153.6^{\circ} \mathrm{C}$. It is noteworthy that the temperature at the outlet of the TEG generator was the lowest of all measurement points-temperatures $t_{2}$ and $t_{3}$ upstream and downstream of the generator had higher values. This was caused by the placement of the thermoresistor out of the axis of the exhaust gas mass flow. Based on the temperature distribution in the TEG generator, it was observed that the thermoelectric modules did not reach the maximum efficiency because the temperature did not exceed $60^{\circ} \mathrm{C}$ on the hot side (Fig. 15). The maximum admissible value of this temperature for the tested thermoelectric modules is $200^{\circ} \mathrm{C}$. An average drop in the temperatures in the TEG generator $\left(t_{\mathrm{gIN}}-t_{\mathrm{gOUT}}\right)$ was $41.4^{\circ} \mathrm{C}$. The temperature of the coolant did not exceed $12^{\circ} \mathrm{C}$.

Low temperature of the exhaust gas in the TEG generator resulted in low values of the voltage $U_{\text {TEG }}$ generated by the thermoelectric modules (Fig. 16). Its average value, from all the modules collectively, was only $33.7 \mathrm{~V}$, whereas the maximum voltage generated by an individual TE module is $12 \mathrm{~V}$. As a consequence, in the entire test, the TE generator produced total $P_{\text {TEG }}=1388.6 \mathrm{~W}$. Due to a low value of the accumulated power of the TE generator, the authors decided to carry out an additional test run at full engine power $(S=2500 \mathrm{rpm}, T=120 \mathrm{Nm})$. At this point, the maximum temperature of the exhaust gas was $t_{\text {gIN }}=301.2^{\circ} \mathrm{C}$, and the average temperature on the hot side of the TE modules was $146.5^{\circ} \mathrm{C}$. This resulted in the production of much higher voltage by the TE generator $\left(U_{\mathrm{TEG}}=\right.$ $132.6 \mathrm{~V})$, which was reflected in the greater maximum power of $P_{\mathrm{TEG}}=135.1 \mathrm{~W}$. With reference to the maximum power obtained in the driving cycle, this was an $85 \%$ increase.

In order to determine the efficiency of the TEG generator in terms of the conversion of exhaust gas thermal energy into electrical energy, one needs to

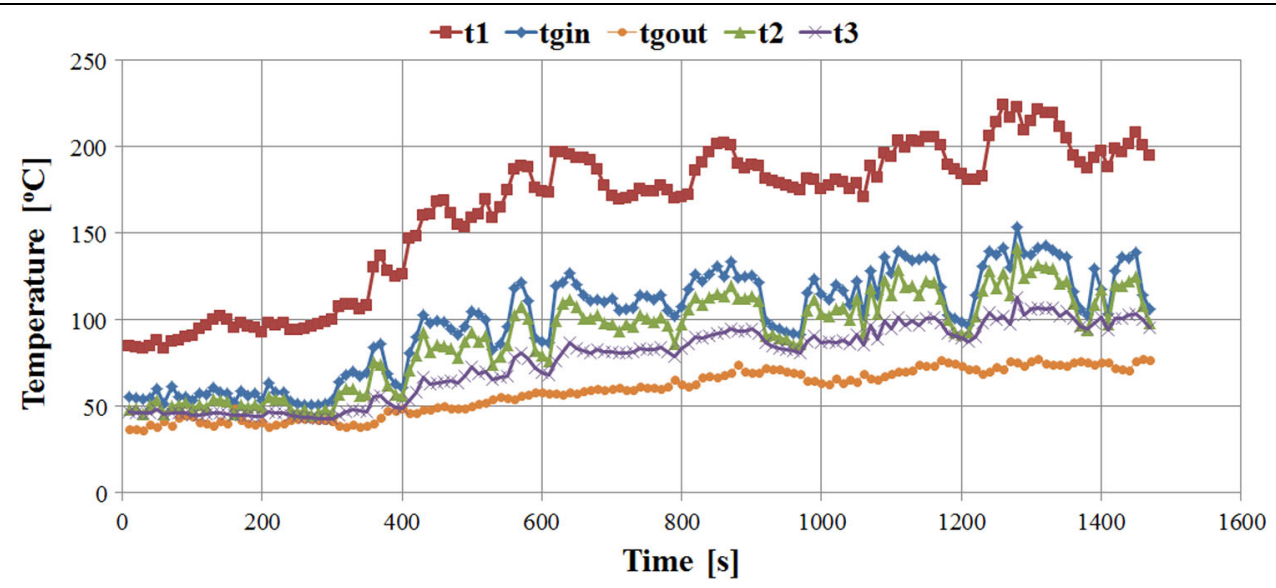

Fig. 14. Temperature distribution in the exhaust gas system fitted with the TEG generator. 
determine the amount of thermal energy from the relationship:

$$
Q_{\mathrm{EXHAUST}}=\underset{\mathrm{EX}}{\dot{m}} \cdot c_{\mathrm{pEX}} \cdot \Delta T_{\mathrm{EX}}
$$

where $Q_{\text {EXHAUst }}$ is exhaust gas energy losses (enthalpy) $(\mathrm{kW}), \dot{m}$ is exhaust gas mass flow $(\mathrm{kg} / \mathrm{s})$, $c_{\text {pex }}$ is the exhaust gas specific heat at a constant pressure $[\mathrm{kJ} /(\mathrm{kg} \mathrm{K})]$, and $\Delta T_{\mathrm{EX}}$ is the exhaust gas temperature $(\mathrm{K})$.

TEG Generator efficiency $\eta_{\mathrm{TEG}}$ was determined from the relationship:

$$
\eta_{\mathrm{TEG}}=\frac{P_{\mathrm{TEG}}}{Q_{\mathrm{EXHAUST}}}
$$

where $\eta_{\mathrm{TEG}}$ is the efficiency of the TEG generator $(\%), P_{\mathrm{TEG}}$ is the TEG generator power (W), and $Q_{\text {EXHAust }}$ is the exhaust gas energy losses (enthalpy) (W).

For the calculations of the thermal energy of the exhaust gas, the following were adopted: temperature $\Delta T_{\mathrm{EX}}$ (difference between the average temperature of the exhaust gas in the TEG generator and ambient temperature), mass flow of exhaust gas $m_{\mathrm{EX}}$ and specific heat $c_{\text {pex }}$ for a given temperature range. Following the specifications provided by the manufacturer, the exhaust flow measurement accuracy is $\pm 2.5 \%$ of the reading or $\pm 1.5 \%$ of the full scale. For obvious reasons, in the tests presented in the paper, the authors did not utilize the entire measurement range of the exhaust gas mass flow meter. In the measurement range, when maximum exhaust gas flow rates were obtained, the maximum error was $10 \mathrm{~kg} / \mathrm{h}$ but this error occurred in $3 \%$ of the entire test cycle. In further parts of the test run, this error oscillated around $1-2 \mathrm{~kg} / \mathrm{h}$. Given the non-linearity of the conversion of thermal energy into electrical, the impact of this error is negligibly low. The maximum efficiency $\eta_{\text {TEG }}$ occurred for low speeds of the reproduced test run and was $1.7 \%$ at its maximum (Fig. 17). As the speed increased, this value dropped to below $0.2 \%$. A reverse situation occurred for power $P_{\mathrm{TEG}}$, whose maximum values were recorded at high speeds of the test run.

The above-presented efficiency $\eta_{\text {TEG }}$ does not allow for the power drop $P_{\text {loss }}$ in the exhaust system

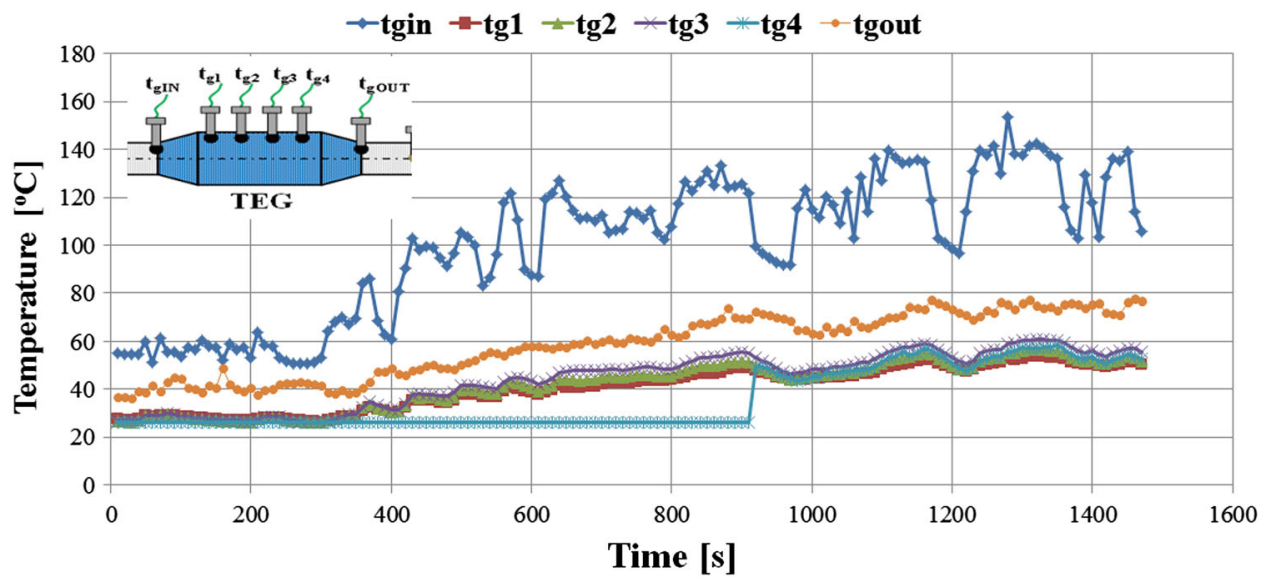

Fig. 15. Temperature distribution in the TEG generator.

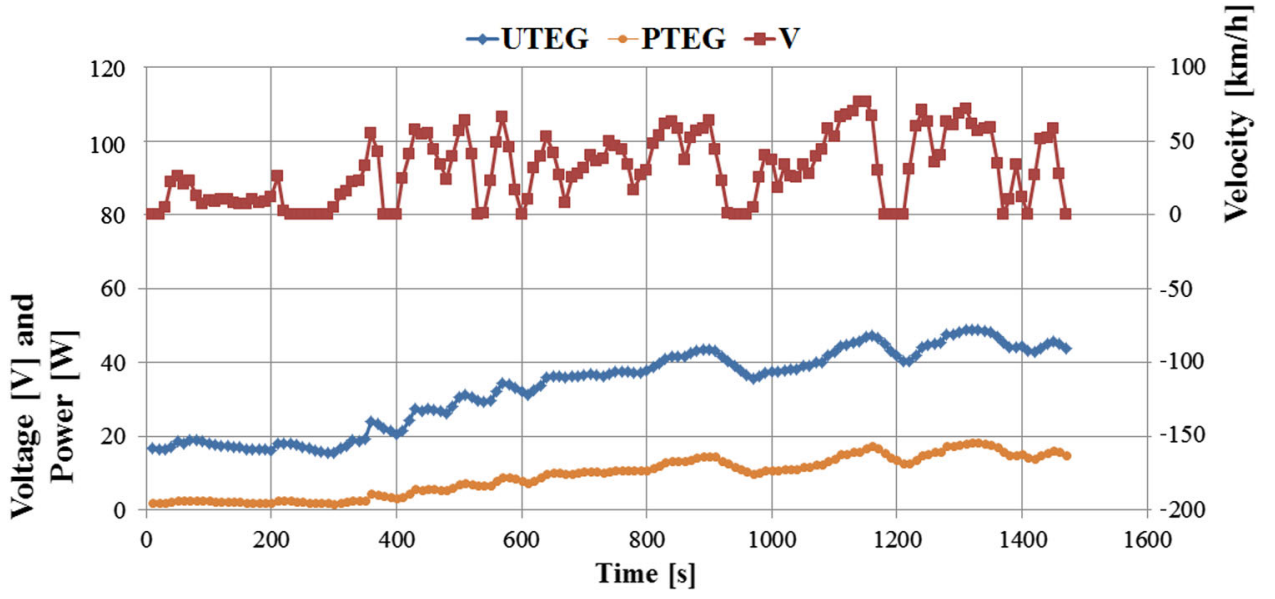

Fig. 16. Voltage and power generated by the TEG generator against vehicle speed. 


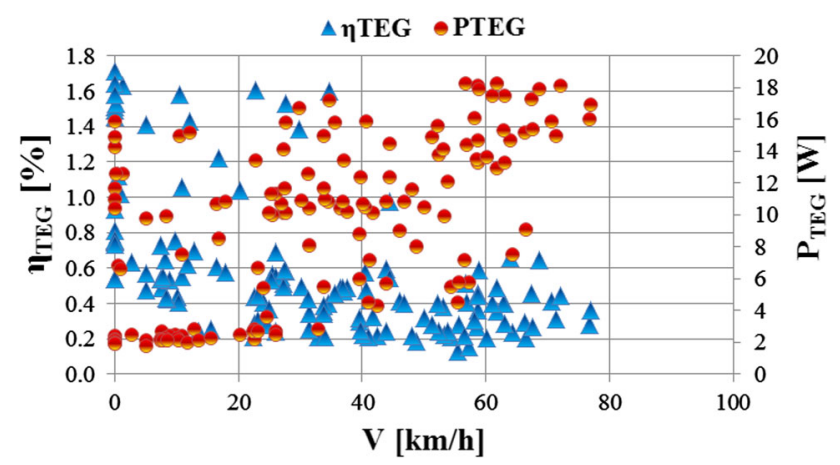

Fig. 17. Power and efficiency of the TEG generator depending on the speed of the reproduced vehicle.

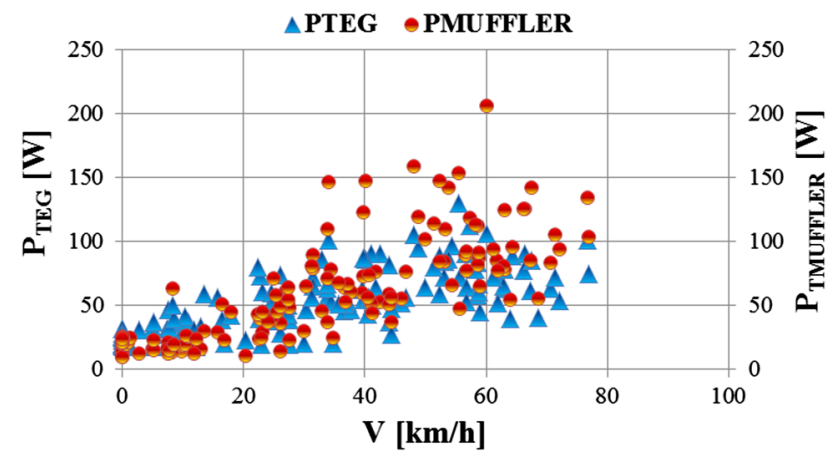

Fig. 18. Power losses in the TEG generator depending on the speed of the reproduced vehicle.

of the tested engine resulting from the fitting of the TEG generator. In order to determine the value $P_{\text {loss }}$ we need to use the relation:

$$
P_{\mathrm{loss}}=\Delta p \cdot \underset{\mathrm{EX}}{\dot{V}}
$$

where $P_{\text {loss }}$ is the power losses (W), and $\Delta p$ is the pressure drop $(\mathrm{Pa})$, and $V$ is the exhaust gas volumetric flow $\left(\mathrm{m}^{3} / \mathrm{s}\right)$.

The TEG generator fitted in the exhaust gas of the tested engine causes a drop of power $P_{\text {loss }}$ in the range 17.4-129.9 W. Referring these values to the power $P_{\mathrm{TEG}}$ obtained in the simulated vehicle, it was observed that they are much higher. A comparison of the power losses between the TEG generator and a standard muffler in the exhaust system has shown that at low speeds the TEG generator generates greater losses (Fig. 18). For medium and high speeds, these losses are comparable.

\section{CONCLUSIONS}

The reproduction of the actual driving cycle on an engine test bed including the entire drivetrain system, design parameters and driver behavior allows the determining of the influence of the application of a TEG generator in the exhaust system on the engine overall efficiency under varied operating conditions. To date, laboratory tests have only been a simulation of individual engine work points that were an approximated reproduction of its actual operating conditions. The performed tests have shown that the application of a TEG generator at the end of the exhaust system did not positively influence the engine overall efficiency. In the reproduced urban and suburban driving conditions, the TEG generator generated a total of $1388.6 \mathrm{~W}$ because, as the analysis of the overall characteristics of the engine operation has shown, it mainly operated under medium and high loads. This was reflected in the thermodynamic parameters of the exhaust gas, particularly in the temperature distribution in the exhaust system. The maximum energy conversion efficiency of the TEG generator (thermal into electrical) was $1.7 \%$. The application of the TEG generator in the exhaust gas of the tested engine also resulted in a decrease of the engine power due to increased flow resistance, whose value was greater than the generated power.

The authors plan to perform similar investigations on a spark ignition engine to assess the influence of the TEG generator on its overall efficiency.

Because the results of the investigations for the diesel engines were unsatisfactory, a new design of the TEG generator has been developed. The new TEG generator has a heat exchanger of better heat transfer efficiency and lower flow resistance compared to that presented in the paper. The design works were primarily based on model research utilizing the methods of numerical fluid mechanics, which led to a successful development of the new TEG generator. Current research is a validation of the new TEG generator. The results of these investigations will be presented in upcoming publications.

\section{OPEN ACCESS}

This article is distributed under the terms of the Creative Commons Attribution License which permits any use, distribution, and reproduction in any medium, provided the original author(s) and the source are credited.

\section{REFERENCES}

1. M. Bajerlein and L. Rymaniak, The reduction of fuel consumption on the example of ecological hybrid buses. Appl. Mech. Mater. 518, 96 (2014).

2. J. Merkisz, P. Fuc, P. Lijewski, and A. Ziolkowski, The onroad exhaust emissions from vehicles fitted with the startstop system. Appl. Mech. Mater. 390, 343 (2013).

3. M.P. Walsh, Global trends in motor vehicle pollution control; a 2011 update. Part 1. Combustion Engines. Silniki Spalinowe 50, 106 (2011).

4. H. Faust, Powertrain Systems of the Future. Engine, transmission and damper systems for downspeeding, downsizing and cylinder deactivation. Schaeffler Symposium Book (Steinhagen: Schaeffler Technologies $\mathrm{GmbH}$ \& Co., 2014), pp. 25-41. 
5. V. Korte, D. Hancock, and H. Blaxill, The Mahle downsized engine as technology demonstrator concept, layout and design. MTZ Worldwide 69, 4 (2008). doi:10.1007/BF03226879.

6. S. Hase, Prospects for TEG Practical Applications, by Physical Evaluation in varied Heat Recovery Technologies (Paper presented at Thermoelectrics Goes Automotive II (Thermoelectrics III), pp. 49-56, 2013).

7. K.T. Wojciechowski, et al., Prototypical thermoelectric generator for waste heat conversion from combustion engines. Combustion Engines 154, 60 (2013). ISSN: 0138-0346.

8. O. Dingel, T. Semper, V. Ambrosius, and J. Seebode, Waste Heat Recovery: What are the Alternatives to the thermoelectric Generator? (Paper presented at Thermoelectrics Goes Automotive II (Thermoelectrics III), 2013), pp. 30-49.

9. L. Aixala, and V. Monnet, Cinclusion of RENOTER Project (Waste Heat Recovery for Trucks and Passenger Cars) (Paper presented at Thermoelectrics Goes Automotive II (Thermoelectrics III), 2013), pp. 241-259.
10. G. Buschamn and C. von Essen, TEGs in Context of Future Drive Concepts (Paper presented at Thermoelectrics Goes Automotive II (Thermoelectrics III), 2013), pp. 1-10.

11. J. Merkisz and P. Fuc, The exhaust emission from light duty vehicles in road test in urban traffic. SAE Int. J. Fuels Lubr. 3, 467 (2010)

12. J. Merkisz, P. Fuc, P. Lijewski, and A. Ziolkowski, The application of nanometric composite materials in a diesel engine in the aspect of improvement of deep bed filtration in a Diesel Particulate Filter (Paper presented at International Congress of FISITA 2012, F2012-A04-031, Bejing, 2012).

13. Vehicle Exhaust Flow Meter Semtech EFM. User's Manual, Document 9510-085, Revision 1.4. Sensors Inc. 2001-2007.

14. ISAC 400 PUMA OPEN V1.5 User's Guide. AVL, AT2775E, Rew. 12 July 2009.

15. SSQ AVL PUMA OPEN 2011 User's Guide. AVL, AT3475E, Rew. 14 April 2011.

16. Thermoelectric module TMG-241-1.4-1.2 SCTB NORD A Ferrotec Group Company. 